\title{
Stress- and Temperature-Dependent Wideband Fluorescence of a Phosphor Composite for Sensing Applications
}

\author{
W. Zhang ${ }^{1,2} \cdot$ Z. Li $^{1} \cdot$ G.W. Baxter ${ }^{2} \cdot$ S.F. Collins ${ }^{2}$
}

Received: 7 December 2015 / Accepted: 19 August 2016/Published online: 25 August 2016

(C) The Author(s) 2016. This article is published with open access at Springerlink.com

\begin{abstract}
As both stress and temperature can alter the lattice of a solid phosphor in a similar way, the dopant rare-earth ion may be used as a probe for either parameter using the same sensing mechanism. Here, a wideband phosphor, YAG:Ce is used as a micro stress sensor at very low magnitudes of compressive stress. The sensing resolution can be smaller than $0.06 \mathrm{~N} / \mathrm{mm}^{2}$ in the range of $0 \sim 1.2 \mathrm{~N} / \mathrm{mm}^{2}$ with a sensitivity of $11 \mathrm{~cm}^{-1} / \mathrm{MPa}$. The sensitivity is about a hundred times larger than the coefficient reported under hydrostatic pressure. The barycenter energy of the emission band of a phosphor can be used to indicate stress and/or temperature change, producing a precision beyond the spectroscopic resolution limit. The sensing functions obtained from the barycenter shift are superior to those from peak shifts. The stress/temperature dependent barycenter shift of the observed fluorescence bands of YAG:Ce were similar in magnitude. Mechanisms involving the nephelauxetic effect, crystal field effect and site symmetry of dopant ions are explored as explanations of the experimental phenomena.
\end{abstract}

Keywords Stress $\cdot$ Temperature $\cdot$ Sensing $\cdot$ Fluorescence

W. Zhang

zdw@ustc.edu

1 Jiangxi Engineering Laboratory for Optoelectronics Testing Technology, Nanchang Hangkong University, Nanchang 330063, China

2 Optical Technology Research Laboratory, Victoria University, P.O. Box 14428, Melbourne, VIC 8001, Australia

\section{Introduction}

It is well known that certain micro-force sensors make use of changes in the fluorescence emitted from specific materials to measure both applied pressure [1, 2] and stress [3]. The changes in fluorescence are often attributed to a shortened bond length between atoms in a strengthened crystal field, resulting in a shift in the associated energy levels. For such sensing applications, a wavelength shift at the intensity maximum of the emission peak(s) of a phosphor is normally used as the sensing element. However, because the scale of the shift is always small, the fluorescence intensity needs to be strong to achieve an acceptable signal-to-noise ratio. Even so, considering that it is desirable that the peak shift should be significant upon applied pressure or stress, there are not many choices for the active material. Ruby $\left(\mathrm{Al}_{2} \mathrm{O}_{3}: \mathrm{Cr}^{3+}\right)$ is a popular active material whose sharp emission peak(s) facilitate distinguishing a slight shift of the peak. The fluorescence of $\mathrm{Cr}^{3+}$ has found wide application in hydrostatic pressure detection in a diamond anvil cell [4] and in residual stress sensing [5]. However, when using the fluorescence method only high pressure or stress (over hundreds MPa) can be retrieved with relatively high precision.

Some rare-earth doped phosphors have very high quantum efficiency, e.g. the well-known yellow phosphor, YAG:Ce. For this phosphor the parity allowed $5 \mathrm{~d}-4 \mathrm{f}$ inter-configurational transitions of $\mathrm{Ce}^{3+}$ are highly sensitive to the change of environmental factors because the d-electrons are in the outer shell. As a consequence the phosphor is known to exhibit pressure-dependent fluorescence which may be attributed to the change of the $\mathrm{Ce}-\mathrm{O}$ bond length [6]. Therefore, the radiative transitions of $\mathrm{Ce}^{3+}$-doped phosphors are expected to be sensitive to changes in stress and/or temperature which lead to similar $\mathrm{Ce}-\mathrm{O}$ bond changes in the lattice of the matrix material. The emission spectrum of YAG:Ce reveals two highly 
overlapping bands. The application of high pressure drives the two bands to shift towards a lower energy with almost the same piezo-spectroscopic coefficient $[6,7]$. Determining the peak position of the resultant broad band is imprecise, and as a consequence, it is difficult to accurately extract shifts in the peak position particularly at a low pressure or under small magnitudes of applied stress. This may explain why YAG:Ce has not been used previously as a probe for applied strain/stress of low magnitude.

The term "barycenter" is most often used in astronomy to describe the location of the center-of-mass of a many-body system. However, it has also come to be used to describe a weighted mean position of fluorescence peaks, particularly, as is the case here, where there is a broad spectral feature masking a number of underlying peaks [8-10]. In this article, it is shown that the barycenter of an emission band functions well as an alternative to the peak wavelength to indicate the stress change for low magnitudes of stress. An extra advantage is that such a sensing method does not require the measurement spectrometer to have a high resolution; an important consideration in keeping instrumental costs and dimensions to a minimum. In this work YAG:Ce has been used to illustrate the sensing technique. The technique described was also applied to temperature sensing, as the same physical effect is evident in the coordination change of $\mathrm{Ce}^{3+}$ caused by either stress or temperature.

\section{Experimental Arrangement}

The sample precursor was a mixture of a YAG:Ce phosphor powder (Keheng $\left.{ }^{\mathrm{TM}} \mathrm{Y} 4454\right)$ and a silicone resin with a mixing ratio of 1:5 by weight. Air bubbles were removed from the mixture under vacuum and then cured in air. The resulting compound was trimmed into a slice $\left(6.0 \times 7.0 \times 1.5 \mathrm{~mm}^{3}\right)$. During the piezo-spectroscopic analysis, compression was applied along the thickness direction. The sample was sandwiched between a glass slide and a push force gauge, as shown schematically in Fig. 1. The resolution of the gauge was $0.25 \mathrm{~N}$, and the measurement range was $0-50 \mathrm{~N}$. For the temperature-dependent test, the phosphor powder was used unadulterated to avoid any possible influence of the resin material. In practice, no notable evidence of the resin's influence on the phosphor fluorescence was found in this work or has been reported elsewhere, while the silicone material is widely used in white LEDs as conformal coating on the wavelengthconverting phosphor(s).

A diode laser with a peak wavelength of $405 \mathrm{~nm}$ was used as the excitation light source. All emission spectra were measured with an AvaSpec-2048TEC-USB2 fiber-optic spectrometer. The finest resolution of the spectrometer is $0.5 \mathrm{~nm}$ at the wavelength $500 \mathrm{~nm}\left(20 \mathrm{~cm}^{-1}\right.$ at the wavenumber $20,000 \mathrm{~cm}^{-1}$ ). A dye filter, located up beam of the spectrometer, removed scattered excitation light from the recorded spectra. The phosphor's structure has been confirmed on a D8ADVANCE X-ray diffractometer, using a $\mathrm{Cu} \mathrm{K} \mathrm{K}_{\alpha} \mathrm{X}$ ray source.

\section{Results and Discussions}

The X-ray diffraction (XRD) pattern of the phosphor powder sample is shown in Fig. 2 and accords with PDF (powder diffraction file) No. 88-2048, confirming that the phosphor has a garnet structure. The resulting emission spectra (Fig. 3) show fluorescence typical of $\mathrm{Ce}^{3+}$-doped YAG. No obvious difference of the spectra features between the powder sample and the composite sample was found. At very low temperature, a spectrometer with fine resolution may directly distinguish two peaks at about $536 \mathrm{~nm}$ and $567 \mathrm{~nm}$, respectively, in the emission spectrum of YAG:Ce [11]. Both emission bands are electron transitions from the lowest $5 \mathrm{~d}$ level to $4 \mathrm{f}$ multiplets $\left({ }^{2} \mathrm{~F}_{7 / 2}\right.$ and $\left.{ }^{2} \mathrm{~F}_{5 / 2}\right)$ of $\mathrm{Ce}^{3+}$.

The two wide fluorescence emission bands of YAG:Ce overlap to such an extent that stress-dependent changes are almost indistinguishable, as is evident in Fig. 3, leading to difficulties in locating precisely any changes to the peak position of the amalgamated band. For example, to observe a pressure-dependent peak shift in the fluorescence emitted from the phosphor, a pressure as great as tens of kilobar (several GPa) is required [7]. Accordingly, a high-resolution spectrometer would be obligatory for the measurement to be successful. When a low level stress is applied to the phosphor, it is not possible to observe any shift in the peak position for the emission spectra, as indicated by the left inset of Fig. 3 . Likewise multi-peak fitting cannot be successfully used to reveal a trend in the relationship between the peak wavelength and the applied stress.

However, there is evidence in the spectra (Fig. 3) corresponding to changes in the applied compressive force. On the high energy side of the merged emission bands of $\mathrm{Ce}^{3+}$, the band edge is observed to be shifting as shown in the right inset of Fig. 3 while on the other side of the band, the successive fluorescence curves essentially overlap each other. This observed shift is normally regarded as being in accordance with the experimental features of the nephelauxetic effect and the change of the crystal field strength $[10,12]$. The expected manifestation of the nephelauxetic effect is for the barycenter energy of a rare-earth ion's energy levels to shift, characterizing a change in the coordination bond length of the ion. In this paper further analysis of the emission spectra is based on the observation of the barycenter shift of the emission band.

The nephelauxetic effect seeks to describe the size of the electron cloud around the central ion which increases due to the transfer of electron density to a bonding molecular orbital. 
Fig. 1 Schematic of the experimental setup for measuring stress-dependent fluorescence

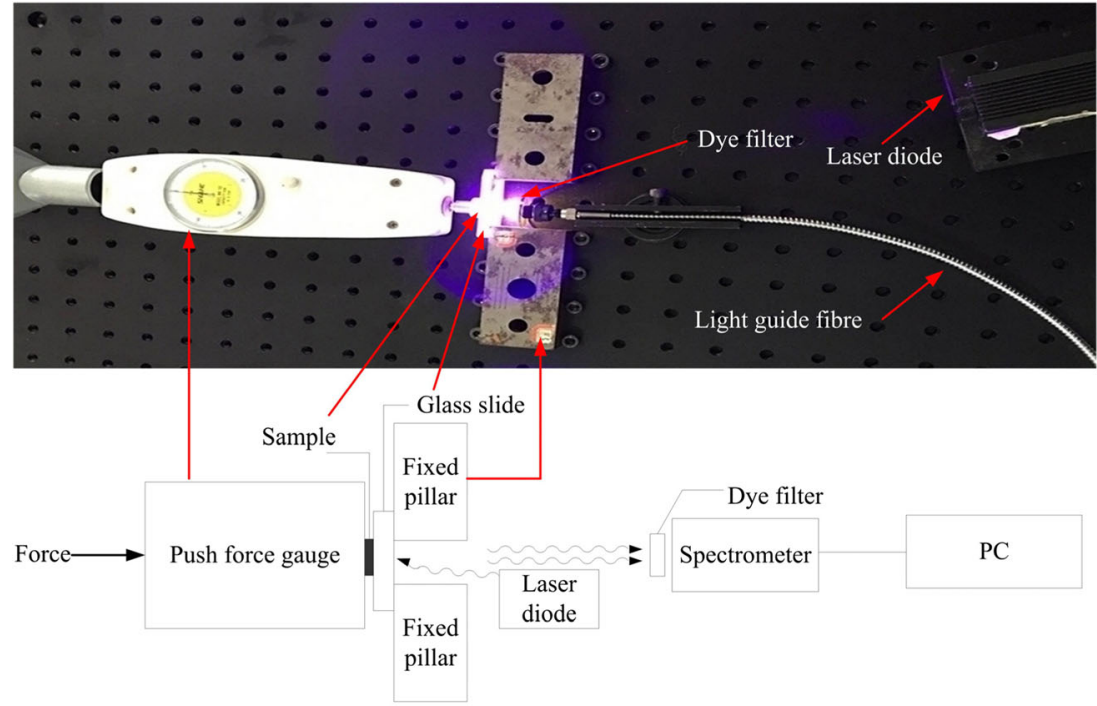

Experimentally the effect can be seen as a shift of the absorption or emission bands caused by an environmental change resulting in changes to the fluorescence spectrum [12]. Spectral changes can therefore be linked to the environmental change and consequently the potential application as a sensing element is realized. For example, for the $4 \mathrm{f}^{\mathrm{n}}-4 \mathrm{f}^{\mathrm{n}}$ transitions of $\mathrm{Eu}^{3+}$, a linear relationship between the barycenter energy and temperature has been reported [9]. As for YAG:Ce, compression onto the phosphor composite makes the average $\mathrm{Ce}-\mathrm{O}$ distance shorter and consequently lower the barycenter energy of the $5 \mathrm{~d}$ levels. Taking into account that the emission relates to the transition from the lowest $5 \mathrm{~d}$ level of $\mathrm{Ce}^{3+}$, a strengthened crystal field will also contribute to a red shift of the emission band barycenter via increasing the splitting of the $5 \mathrm{~d}$ levels.

Considering that the $4 \mathrm{f}$ electrons are shielded by the $5 \mathrm{~d}$ electron shell, the ground $4 \mathrm{f}$ level(s) of $\mathrm{Ce}^{3+}$ is not expected to be greatly affected by environmental changes. Evidence for this lies in the fact that the two emission bands stay merged as

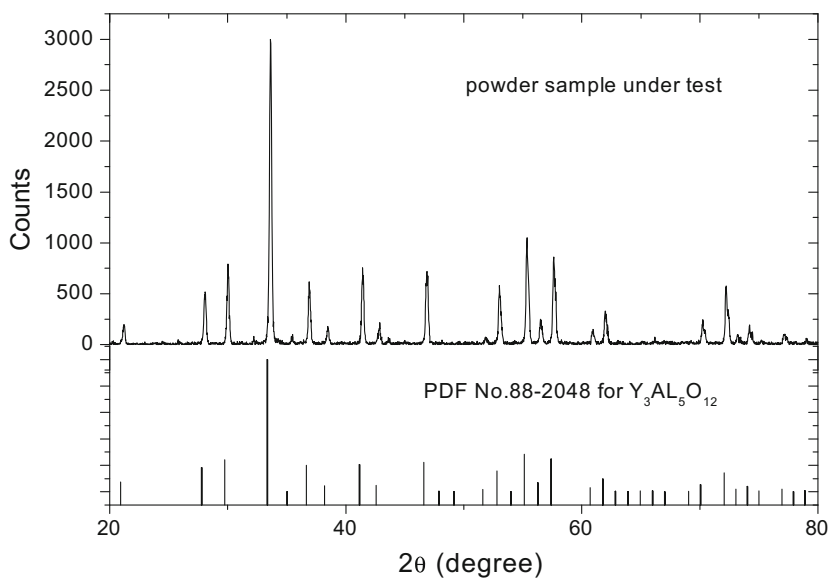

Fig. 2 X-ray diffraction pattern of the YAG: Ce phosphor powder one observable band, not separating under normal pressure [6, 7] or stress (Fig. 3). In this circumstance the separation width of the two sub-bands may be equated to the splitting width of the $4 \mathrm{f}$ ground level, which is determined by the crystal field strength. As mentioned above, multi-peak fitting cannot produce a self-consistent monotonic change of the separation width as the applied compressive force is increased, and such an analysis was not performed in this work. The compressiondependent shift of the $\mathrm{Ce}^{3+}$ emission band barycenter is shown in Fig. 4 and its magnitude is observed to be nearly equal to the shift of the $5 \mathrm{~d}$ lowest level of $\mathrm{Ce}^{3+}$. Hence by determining the shift in the barycenter of the emission bands, at various compression levels, the effect of compression becomes clearly observable in contrast to the very small changes to the peak positions of the two merged bands of $\mathrm{Ce}^{3+}$ emission. In fact, as shown in Fig. 4 and also in the left inset of Fig. 3, the fluctuations in intensity made any shift in the peak position almost indistinguishable, even though the signal-to-noise ratio of the spectra is relatively high at about 700:1 (calculated with the peak-to-peak baseline noise and the fluorescence peak intensity from the real spectra in this study).

Figure 4 suggests a near linear sensing region for part of the range of applied compressive force. The line gives a piezospectroscopic coefficient of about $11 \mathrm{~cm}^{-1} / \mathrm{MPa}$ (compression over the sample area $\sim 42 \mathrm{~mm}^{2}$ ). This shift coefficient is of the order of one hundred times larger than the reported hydrostatic pressure coefficient of the YAG:Ce emission peaks [7]. In addition the direction of the shift is the reverse of what is expected. As for the first datum point which is free of external stress loading, neither the linear nor the polynomial fitting curve are able to accommodate it, most probably implying the existence of residual stress in the composite sample. The measured resolution is less than $0.06 \mathrm{~N} / \mathrm{mm}^{2}(0.06 \mathrm{MPa}, 2.5 \mathrm{~N}$ over $42 \mathrm{~mm}^{2}$ surface area), with a barycenter energy shift of $0.6 \mathrm{~cm}^{-1}$. This is better than the spectrometer resolution 
Fig. 3 Emission spectra of the composite sample under varying magnitude of compressive force. The spectra have been normalized to the peak intensity. Selected portions are magnified in the insets

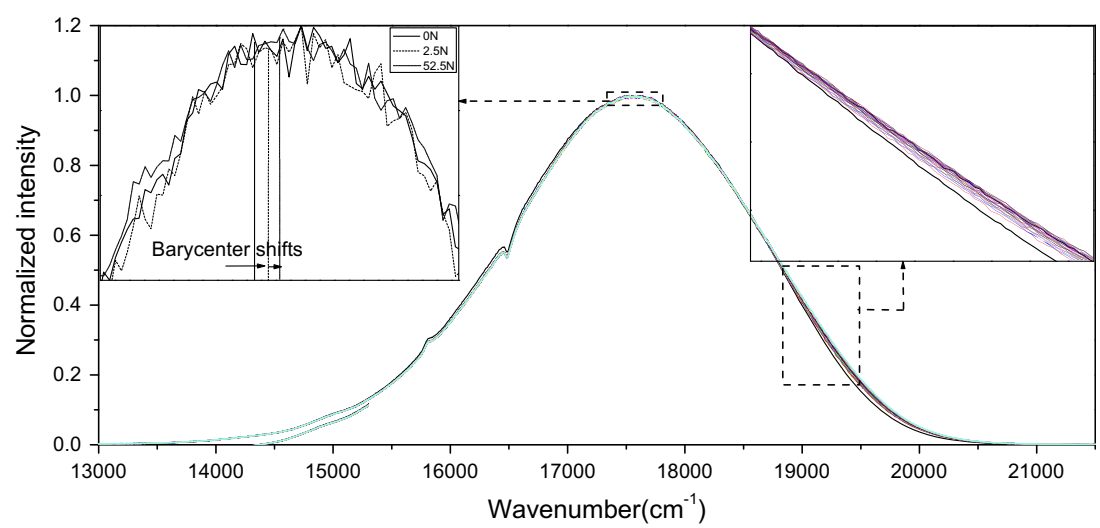

$\left(20 \mathrm{~cm}^{-1}\right.$ at $\left.500 \mathrm{~nm}\right)$. That is to say, the sensing function performs well for applications of stress measurement at a very low magnitude of stress. Further, the sensing phenomenon is contrary to the prediction of the nephelauxetic effect and crystal field theory which would expect to present a red shift.

For practical applications, it is not essential that the measurement be related to the nephelauxetic effect when retrieving and/or applying the barycenter of fluorescence peaks/ bands. Notwithstanding the physical meaning or any ambiguity of the stress sensing mechanism, the above experimental analysis of stress-dependent spectra has shown the advantages of using the barycenter technique. While the method is compatible with traditional peak-locating operations (as both characterize the band position) the resolution of the barycenter technique was shown to be superior to the resolution limit of the spectrometer used in the measurement. Therefore a very small stress magnitude is able to be distinguished by using the barycenter technique. To our best knowledge, it is the first time that a low stress (several $\mathrm{KPa}$ ) has been precisely measured via a fluorescence method. Particularly there have not been any similar reports on the stress-dependent properties of $\mathrm{Ce}^{3+}$-doped garnet family of materials.

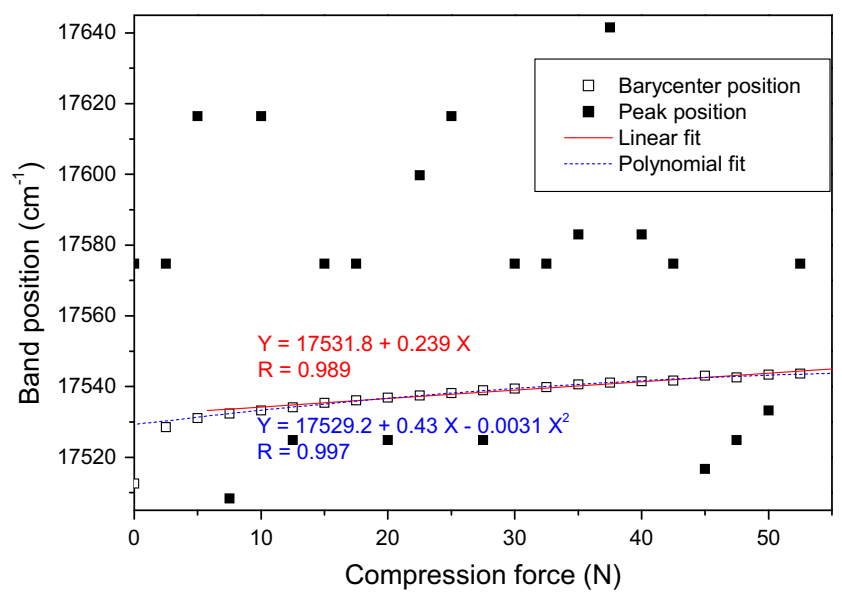

Fig. 4 Barycenter energy of the emission band of the composite sample under varying compressive force
The unpredicted shift direction remains unexplained. Considering that the lanthanide-ligand distance is both temperature- and stress-dependent, an investigation of the temperature-dependent fluorescence (Fig. 5) was undertaken in an attempt to clarify the piezo-spectroscopic ambiguity. Any change in the barycenter energy caused by different environmental parameters can be considered as having the same coordination circumstance dependence [9].

Consistent with the unpredicted result with applied stress reported above, Fig. 5 shows temperature related shifts in the peak position opposite in direction to that expected by recourse to the nephelauxetic effect and crystal field theory. A thermally expanded lattice constant means weaker electron cloud repulsion and also a weakened crystal field. These effects should raise the lowest $5 \mathrm{~d}$ level of $\mathrm{Ce}^{3+}$, showing a blueshifted emission band in direction contrast to the experimental result.

By way of exploration, there are other operations on the material that can vary the lattice constant and consequently influence the resultant fluorescence phenomena potentially in a way that is comparable with our observations. Such lattice expansion or shrinkage could be a result of the heat treatment temperature on sample phosphors $[13,14]$ or an effect of the nanoscale sample particle size $[15,16]$. Alternatively there may have been $\mathrm{Y}^{3+}$ substitution by $\mathrm{Gd}^{3+}[17-23]$ or $\mathrm{Tb}^{3+}$ [24] or again $\mathrm{Lu}^{3+}[25,26]$; perhaps there was a different doping concentration of $\mathrm{Ce}^{3+}$ [27-29]. Even though these articles reported very similar wavelength shifts of the emission band maximum, the mechanism of such a lattice effect on $\mathrm{Ce}^{3+}$ emission were ambiguously allocated to strengthened or weakened crystal field in most of the articles. Tanner et al. have pointed out similar conflicts in various publications when they reassigned energy levels of YAG:Ce [30].

There are more conjectures that may be related to the shift of the emission of YAG:Ce. The garnet structure possesses several types of positions for the accommodation of dopant ions [31, 32]. Dopant pairs/clusters can possibly produce lower energy sites in the $\mathrm{Lu}_{3} \mathrm{Al}_{5} \mathrm{O}_{12}$ matrix and then the emission spectra show a strong red shift [33]. However, it is common 
Fig. 5 Temperature-dependent emission spectra of the phosphor powder sample, normalized to the peak intensity. A selected portion is magnified in the inset

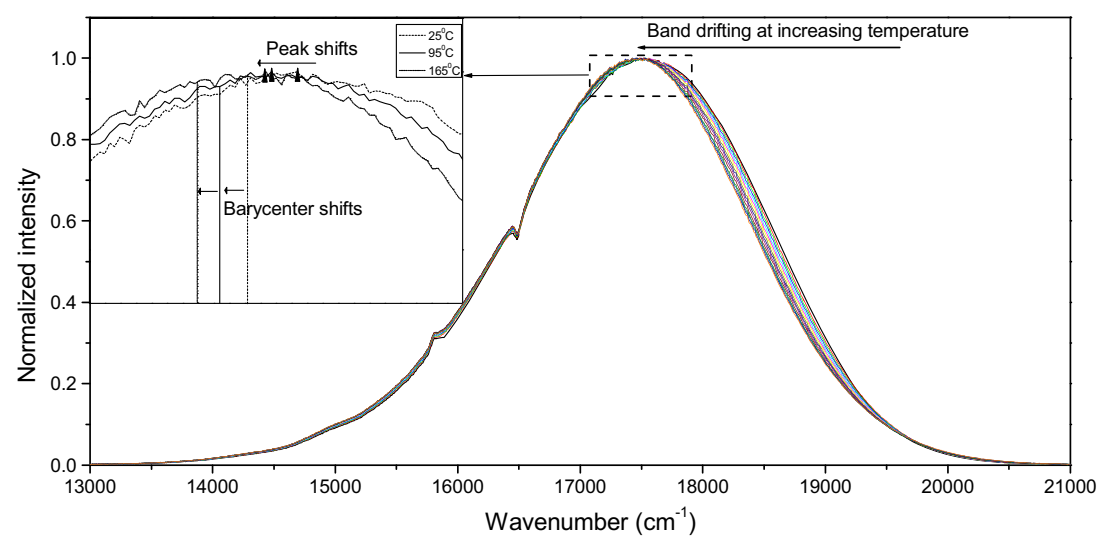

that $\mathrm{Ce}^{3+}$ replaces $\mathrm{Y}^{3+}$ in the YAG material but not in other positions if the synthesizing temperature is not too high [30, 34]. Moreover, in a certain material with fixed doping concentration, the dopant ions are not able to re-distribute reversibly under the influence of temperature or stress. Then the above findings do not reveal the major mechanism of the observations reported in this work. Neither do other speculations, such as re-absorption processes [35] or a modified crystal field theory [36]. Considering that the major mechanism of the observed shifts should be of the coordination circumstance of the central $\mathrm{Ce}^{3+}$ ion and nothing else, it was determined that an altered site symmetry of the central ion should be taken into account.

Andriessen and co-workers have shown that lattice deformation leads to a significant shift in $\mathrm{Ce}^{3+}$ ion fluorescence wavelength [37]. More reports [38-41] suggest that deviation from cubic symmetry around the $\mathrm{Ce}^{3+}$ ion results in an increase in the maximum emission wavelength, while yttrium aluminum garnet provides a distorted cubic local circumstance to the dopant [34]. Besides the radius of the dopant ion, environmental parameters such as temperature and stress can produce a similar distortion. Anisotropic thermal expansion of the matrix YAG material drives the $\mathrm{Ce}-\mathrm{O}$ cell microstructure distortion. On the other hand, stress can shape the microstructure to achieve a higher or lower symmetry, depending on the stress direction and scale. On average, for a phosphor powder-resin composite, compression should slightly diminish the difference of $\mathrm{Ce}-\mathrm{O}$ distances, meaning an increasing symmetry. Lower point symmetry of $\mathrm{Ce}^{3+}$ allows a significant modification of the separation gap between the lowest two $5 \mathrm{~d}$ levels, namely the two ${ }^{2} \mathrm{E}$ levels of $\mathrm{Ce}^{3+}$ in a garnet structure [38-41]. Both the temperature influence and the compression effect of YAG:Ce fluorescence can imply different distortions of the solid lattice. The distortion influence can be so important that the crystal field effect is more than being compensated. Only when the change of the coordination circumstance around $\mathrm{Ce}^{3+}$ is isotropic, e.g. under a hydrostatic pressure, the coordination symmetry issue is no longer effective. This consideration can explain why the observed stress and temperature effects in the present experiment are different to those reported in previous publications $[6,7]$, whilst the phenomena is more complicated than the prediction of the nephelauxetic effect, crystal field theory, and the Jahn-Teller effect, e.g. the remaining problem in multi-peak fitting.

As well as the usage of the phosphor-resin composite as a stress gauge, the temperature-dependent monotonic shift of emission band (Fig. 5) can be used for temperature sensing. Influences of temperature on the position of both the band peak and barycenter energy are shown in Fig. 6. The trends in the data for each method of analysis are similar; however the barycenter shifts indicate a much smaller uncertainty in the determination of the sensitivity $\left((-0.55 \pm 0.03) \mathrm{cm}^{-1} /{ }^{\circ} \mathrm{C}\right.$ compared with that for the position of the band peak $(-0.86 \pm$ $\left.0.17) \mathrm{cm}^{-1} /{ }^{\circ} \mathrm{C}\right)$. The form of barycenter sensing line is well defined but not yet understood. To enable a comparison with the peak center technique, the barycenter sensing line has been fitted with a linear function. The correlation coefficient for the linear fitting of the barycenter sensing is -0.98 , suggesting that the linear fit is acceptable and applicable. This improvement in measurement precision is a consequence of the

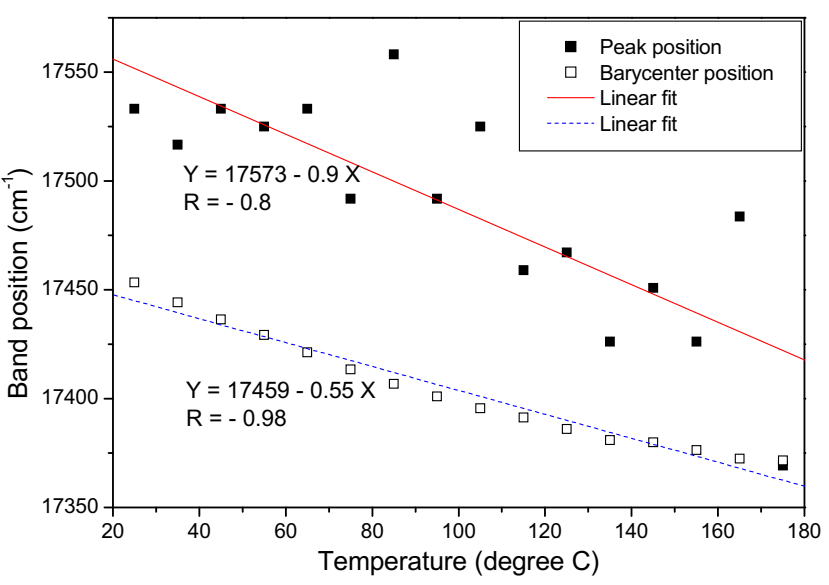

Fig. 6 The temperature-dependent position of peak energy (solid line) and barycenter energy (dashed line) of the powder phosphor's emission band 
analyzing methodology itself as when determining the barycenter energy, the total area of the emission band is integrated first and then the barycenter position divides the area equally. In this way the operation of integration eliminates the random noise of the measurement.

In this article, a shift in the emission band barycenter of $\mathrm{Ce}^{3+}$ indicates more than the change of $\mathrm{Ce}-\mathrm{O}$ distance, either in the stress effect or temperature-dependent spectra. Reasonably, we may look forward to more sensing applications of the barycenter method in other situations with or without change of point symmetry at the active ion position.

\section{Conclusions}

In conclusion, this article has demonstrated that fluorescent materials, in particular YAG:Ce, are available for stress and temperature sensing by carefully monitoring the position of the emission band(s). Sensing functions have been retrieved successfully and beyond the expectation of normal peak analysis when the barycenter energy was employed to characterize the band position. The barycenter energy of emission band(s) can be used as a sensing signal, while the actual operation of a practical device does not require consideration of the underlying physical effect. The barycenter energy provides a relatively fine resolution and high precision to the sensing method. This result is important for sensing applications at low pressure/stress levels in contrast to the "classic" peak shift method that has already proven to be successful for relatively higher stress amplitudes.

Acknowledgments This work is supported by the National Natural Science Foundation of China (Grants No. 61167007 and 11162014), the Aeronautical Science Foundation of China (Grant No. 2012ZD56007), the China Scholarship Council (File No. 201308360026), and the Program for Supporting Young Scientists of Jiangxi Province (Grant No. 20153BCB23037).

Open Access This article is distributed under the terms of the Creative Commons Attribution 4.0 International License (http:// creativecommons.org/licenses/by/4.0/), which permits unrestricted use, distribution, and reproduction in any medium, provided you give appropriate credit to the original author(s) and the source, provide a link to the Creative Commons license, and indicate if changes were made.

\section{References}

1. Wittlin A, Przybylińska H, Berkowski M et al (2015) Ambient and high pressure spectroscopy of $\mathrm{Ce}^{3+}$ doped yttrium gallium garnet. Opt Mater Express 5:1868-1880

2. Grinberg M (2011) Excited states dynamics under high pressure in lanthanide-doped solids. J Lumin 131:433-437

3. Lipkin DM, Clarke DR (1996) Measurement of the stress in oxide scales formed by oxidation of alumina-forming alloys. Oxid Met 45(3/4):267-280
4. Syassen K (2008) Ruby under pressure. High Pressure Res 28:75126

5. Ma Q, Clarke DR (1993) Stress measurement in single-crystal and polycrystalline ceramics using their optical fluorescence. J Am Ceram Soc 76:1433-1440

6. Grinberg M (2006) High pressure spectroscopy of rare earth ions doped crystals - new results. Opt Mater 28:26-34

7. Barzowska J, Grinberg M, Tsuboi T (2003) High pressure spectroscopy of Ce doped YAG crystal. Radiat Eff Defects Solids 158:3947

8. Antic-Fidancev E (2000) Simple way to test the validity of ${ }^{2 S+1} L_{J}$ barycenters of rare earth ions (e.g. $4 \mathrm{f}^{2}, 4 \mathrm{f}^{3}$ and $4 \mathrm{f}^{6}$ configurations). $\mathrm{J}$ Alloys Compd 300-301:2-10

9. Zhang WW, Yin M, He XD, Gao YQ (2011) Size dependent luminescence of nanocrystalline $\mathrm{Y}_{2} \mathrm{O}_{3}: \mathrm{Eu}$ and connection to temperature stimulus. J Alloys Compd 509(8):3613-3616

10. Burdick GW, Reid MF (2007) $4 \mathrm{f}^{\mathrm{n}}-4 \mathrm{f}^{\mathrm{n}-1} 5 \mathrm{~d}$ transitions. In: Gschneidner Jr. KA, Bünzli J-CG, Pecharsky VK (Eds.) Handbook on the Physics and Chemistry of Rare Earths, Elsevier B 37:80

11. Zorenko Y, Voznyak T, Gorbenko V et al (2011) Luminescence properties of $\mathrm{Y}_{3} \mathrm{Al}_{5} \mathrm{O}_{12}$ : Ce nanoceramics. J Lumin 131:17-21

12. Görller-Walrand C, Binnemans K (1996) Rationalization of crystalfield parametrization, in: Gschneidner Jr. KA, Eyring L (Eds.) Handbook on the Physics and Chemistry of Rare Earths. Elsevier Sci 23:163-164

13. Xia G, Zhou S, Zhang J, Xu J (2005) Structural and optical properties of YAG: $\mathrm{Ce}^{3+}$ phosphors by sol-gel combustion method. J Cryst Growth 279:357-362

14. Purwanto A, Wang WN, Ogi T et al (2008) High luminance YAG: Ce nanoparticles fabricated from urea added aqueous precursor by flame process. J Alloys Compd 463:350-357

15. Pazik R, Gluchowski P, Hreniak D et al (2008) Fabrication and luminescence studies of $\mathrm{Ce}: \mathrm{Y}_{3} \mathrm{Al}_{5} \mathrm{O}_{12}$ transparent nanoceramic. Opt Mater 30:714-718

16. Zhang K, Hu WB, Wu YT, Liu HZ (2008) Synthesis, luminescence, and effect of heat treatment on the properties of $\mathrm{Y}_{3} \mathrm{Al}_{5} \mathrm{O}_{12}$ :Ce phosphor. Inorg Mater 44:1218-1223

17. Pan Y, Wu M, Su Q (2004) Tailored photoluminescence of YAG:Ce phosphor through various methods. J Phys Chem Solids 65:845850

18. Pan YX, Wang W, Liu GK et al (2009) Correlation between structure variation and luminescence red shift in YAG:Ce. J Alloys Compd 488:638-642

19. Shi H, Zhu C, Huang J et al (2014) Luminescence properties of YAG:Ce, Gd phosphors synthesized under vacuum condition and their white LED performances. Opt Mater Express 4:649-655

20. Li X, Li JG, Xiu Z et al (2010) Effects of $\mathrm{Gd}^{3+}$ substitution on the fabrication of transparent $\left(\mathrm{Y}_{1-\mathrm{x}} \mathrm{Gd}_{\mathrm{x}}\right)_{3} \mathrm{Al}_{5} \mathrm{O}_{12}$ ceramics. J Am Ceram Soc 93:2229-2235

21. Fujita S, Sakamoto A, Tanabe S (2008) Luminescence characteristics of YAG glass-ceramic phosphor for white LED. IEEE J Sel Top Quant Electr 14:1387-1391

22. Shao Q, Li H, Dong Y et al (2010) Temperature-dependent photoluminescence studies on $\mathrm{Y}_{2.93-\mathrm{x}} \mathrm{Ln}_{\mathrm{x}} \mathrm{Al}_{5} \mathrm{O}_{12}: \mathrm{Ce}_{0.07}(\mathrm{Ln}=\mathrm{Gd}$, La) phosphors for white LEDs application. J Alloys Compd 498: 199-202

23. Li K, Shen C (2012) White LED based on nano-YAG:Ce3+/YAG: Ce3+, Gd3+ hybrid phosphors. Optik 123:621-623

24. Dotsenko VP, Berezovskaya IV, Zubar EV et al (2013) Synthesis and luminescent study of $\mathrm{Ce}^{3+}$-doped terbium-yttrium aluminum garnet. J Alloys Compd 550:159-163

25. Praveena R, Shi L, Jang KH et al (2011) Sol-gel synthesis and thermal stability of luminescence of $\mathrm{Lu}_{3} \mathrm{Al}_{5} \mathrm{O}_{12}: \mathrm{Ce}^{3+}$ nano-garnet. J Alloys Compd 509:859-863 
26. Yang H, Lee DK, Kim YS (2009) Spectral variations of nano-sized $\mathrm{Y}_{3} \mathrm{Al}_{5} \mathrm{O}_{12}$ :Ce phosphors via codoping/substitution and their white LED characteristics. Mater Chem Phys 114:665-669

27. Chung EJ, Masaki T, Song YH et al (2013) Enhancement of thermal quenching properties of a yellow-emitting $\mathrm{SiO}_{2}$-coated $\mathrm{Y}_{3} \mathrm{Al}_{5} \mathrm{O}_{12}: \mathrm{Ce}^{3+}$ phosphor for white light-emitting diode applications. Phys Scr T157:014012

28. Haranath D, Chander H, Sharma P, Singh S (2006) Enhanced luminescence of $\mathrm{Y}_{3} \mathrm{Al}_{5} \mathrm{O}_{12}: \mathrm{Ce}^{3+}$ nanophosphor for white lightemitting diodes. Appl Phys Lett 89:173118

29. Wei N, Lu T, Li F et al (2012) Transparent Ce: $\mathrm{Y}_{3} \mathrm{Al}_{5} \mathrm{O}_{12}$ ceramic phosphors for white light-emitting diodes. Appl Phys Lett 101: 061902

30. Tanner PA, Fu L, Ning L et al (2007) Soft synthesis and vacuum ultraviolet spectra of YAG: $\mathrm{Ce}^{3+}$ nanocrystals: reassignment of $\mathrm{Ce}^{3+}$ energy levels. J Phys Condens Matter 19:216213

31. Lupei A, Lupei V, Gheorghe C et al (2014) Multicenters in $\mathrm{Ce}^{3+}$ visible emission of YAG ceramics. Opt Mater 37:727-733

32. Speghini A, Piccinelli F, Bettinelli M (2011) Synthesis, characterization and luminescence spectroscopy of oxide nanopowders activated with trivalent lanthanide ions: the garnet family. Opt Mater 33:247-257

33. Setlur AA, Srivastava AM (2007) On the relationship between emission color and $\mathrm{Ce}^{3+}$ concentration in garnet phosphors. Opt Mater 29:1647-1652
34. Przybylińska H, Ma CG, Brik MG et al (2013) Electronic structure of $\mathrm{Ce}^{3+}$ multicenters in yttrium aluminum garnets. Appl Phys Lett 102:241112

35. Katelnikovas A, Plewa J, Dutczak D et al (2012) Synthesis and optical properties of green emitting garnet phosphors for phosphor-converted light emitting diodes. Opt Mater 34:11951201

36. Kim KM, Ryu JH (2013) Synthesis of $\mathrm{Y}_{3} \mathrm{Al}_{5} \mathrm{O}_{12}: \mathrm{Ce}^{3+}$ colloidal nanocrystals by pulsed laser ablation and their luminescent properties. J Alloys Compd 576:195-200

37. Andriessen J, van der Kolk E, Dorenbos P (2007) Lattice relaxation study of the $4 \mathrm{f}-5 \mathrm{~d}$ excitation of $\mathrm{Ce}^{3+}$-doped $\mathrm{LaCl}_{3}, \mathrm{LaBr}_{3}$, and $\mathrm{NaLaF}_{4}$ : Stokes shift by pseudo Jahn-Teller effect. Phys Rev B 76:075124

38. Wu JL, Gundiah G, Cheetham AK (2007) Structure-property correlations in Ce-doped garnet phosphors for use in solid state lighting. Chem Phys Lett 441:250-254

39. Hansel R, Allison S, Walker G (2010) Temperature-dependent luminescence of gallium-substituted YAG:Ce. J Mater Sci 45:146150

40. Birkel A, Denault KA, George NC et al (2012) Rapid microwave preparation of highly efficient $\mathrm{Ce}^{3+}$-substituted garnet phosphors for solid state white lighting. Chem Mater 24:1198-1204

41. Shao Q, Dong Y, Jiang J et al (2011) Temperature-dependent photoluminescence properties of $(\mathrm{Y}, \mathrm{Lu}) 3 \mathrm{Al}$ O12:Ce3+ phosphors for white LEDs applications. J Lumin 131:1013-1015 